\title{
REVIEW
}

\section{The dual role of ubiquitin-like protein Urm1 as a protein modifier and sulfur carrier}

\author{
Fengbin Wang, Meiruo Liu, Rui Qiu, Chaoneng $\mathrm{Ji}^{\bowtie}$ \\ Institute of Genetics, Fudan University, Shanghai 200433, China \\ $凶$ Correspondence: chnji@fudan.edu.cn \\ Received June 16, 2011 Accepted June 29, 2011
}

\begin{abstract}
The ubiquitin-related modifier Urm1 can be covalently conjugated to lysine residues of other proteins, such as yeast Ahp1 and human MOCS3, through a mechanism involving the E1-like protein Uba4 (MOCS3 in humans). Similar to ubiquitination, urmylation requires a thioester intermediate and forms isopeptide bonds between Urm1 and its substrates. In addition, the urmylation process can be significantly enhanced by oxidative stress. Recent findings have demonstrated that Urm1 also acts as a sulfur carrier in the thiolation of eukaryotic tRNA via a mechanism that requires the formation of a thiocarboxylated Urm1. This role is very similar to that of prokaryotic sulfur carriers such as MoaD and ThiS. Evidence strongly supports the hypothesis that Urm1 is the molecular fossil in the evolutionary link between prokaryotic sulfur carriers and eukaryotic ubiquitin-like proteins. In the present review, we discuss the dual role of Urm1 in protein and tRNA modification.
\end{abstract}

KEYWORDS Urm1 system, tRNA modification, Ub-like protein modification

\section{INTRODUCTION}

Ubiquitin and ubiquitin-like proteins (UBLs) are small posttranslational modifiers that regulate a multitude of cellular processes by covalently conjugating to target substrates (Ulrich, 2002; Pickart and Fushman, 2004; Sun and Chen, 2004). UBLs are characterized by a conserved C-terminal diglycine and a core $\beta$-grasp-fold structural motif (Furukawa et al., 2000). Known and putative UBLs are listed in Table 1 (Hochstrasser, 2009; Humbard et al., 2010; Miranda et al., 2011). Although not all UBLs have distinct sequence identities with ubiquitin, they seem to share a similar cascaded enzyme reaction pathway. In the presence of ATP, the C-terminal diglycine motif of UBLs is activated by adenylation, which is catalyzed by the activating enzyme E1 and subsequently forms a high-energy thioester bond with the E1 catalytic cysteine (Ciechanover et al., 1981; Haas et al., 1982). Afterward, activated UBLs are transferred to the conjugating enzyme E2 via transthioesterification, and finally form a covalent isopeptide bond with the $\varepsilon$-amine of the lysine residues of their respective protein substrates, with the help of the ligase E3 (Fig. 1) (Hershko et al., 2000; Pedrioli et al., 2008).

The eukaryotic ubiquitin-related modifier (Urm1) was discovered in 2000 via BLAST analysis of several prokaryotic sulfur carrier proteins such as MoaD and ThiS (Furukawa et al., 2000). Compared with other UBLs, Urm1 shows significantly higher sequence identities with sulfur carriers. In Escherichia coli, MoaD and ThiS (Fig. 1) play crucial roles in biosynthetic pathways, with MoaD being involved in molybdenum cofactor (Moco) biosynthesis (Lake et al., 2001) and ThiS in thiamin biosynthesis (Wang et al., 2001). In contrast to the pathways of eukaryotic UBLs, prokaryotic sulfur carriers diverge after the initial ATP-dependent adenylation activation by forming an acyl disulfide bond with their E1s. As a consequence, a thiocarboxylate is formed to provide sulfur for subsequent reactions (Xi et al., 2001; lyer et al., 2006). Since 2008, several independent groups have described the novel function of Urm1 as a sulfur carrier in the 2-thiolation modification of 5-methoxycarbonylmethyl-2thiouridine $\left(\mathrm{mcm}^{5} \mathrm{~s}^{2} \mathrm{U}\right)$ (Fig. 2B) (Huang et al., 2008; Nakai et al., 2008; Schlieker et al., 2008; Leidel et al., 2009; Noma et al., 2009). Results in these studies strongly suggest that Urm1 activation is highly similar to those of prokaryotic sulfur carrier proteins. Urm1 forms an acyl disulfide bond with its $\mathrm{E} 1$ (Uba4 in budding yeast, MOCS3 in humans), leading to the formation of a thiocarboxylated Urm1 (Fig. 1) (Schmitz et al., 2008). 
Table 1 Known and putative UBLs and their activating and conjugating enzymes

\begin{tabular}{|c|c|c|c|c|}
\hline $\mathrm{UBL}^{*}$ & Identity with Ub (\%) & $\mathrm{E} 1^{*}$ & $\mathrm{E} 2^{*}$ & Comments on UBL \\
\hline \multicolumn{5}{|l|}{ Known UBLs } \\
\hline Ubiquitin & 100 & Uba1 (UBA6) & Many & Precursors encoded by multiple genes \\
\hline Rub1 (NEDD8) & 55 & Uba3-Ula1 & Ubc12 & Substrates are cullins and p53 \\
\hline FUBI & 38 & $\mathrm{NI}$ & $\mathrm{NI}$ & Derived from a ribosomal-protein precursor \\
\hline FAT10 & 32 and $40^{\dagger}$ & UBA6 & $\mathrm{NI}$ & Contains a $\beta$-grasp fold, substrates unknown \\
\hline ISG15 & 32 and $37^{\dagger}$ & UBE1L & UBCH8 & Production induced by type I interferons \\
\hline $\begin{array}{l}\text { Smt3 (SUMO1, } \\
\text { SUMO2, SUMO3) }\end{array}$ & 18 & Uba2-Aos1 & Ubc9 & $\begin{array}{l}\text { SUMO encoded by } 3-4 \text { genes in vertebrates, } \\
\text { depending on the species }\end{array}$ \\
\hline Atg8 & ND & $\operatorname{Atg} 7$ & Atg3 & $\begin{array}{l}\text { Three known isoforms in humans } \\
\text { Contains a } \beta \text {-grasp fold }\end{array}$ \\
\hline Atg12 & ND & Atg7 & Atg10 & $\sim 20 \%$ identical to Atg8 \\
\hline Urm1 & ND & Uba4 & $\mathrm{NI}$ & $\begin{array}{l}\text { Involved in tRNA modification } \\
\text { Contains a } \beta \text {-grasp fold }\end{array}$ \\
\hline UFM1 & ND & UBA5 & UFC1 & Contains a $\beta$-grasp fold \\
\hline SAMP1, SAMP2 & ND & UbaA & $\mathrm{NI}$ & $\begin{array}{l}\text { Archaeal modifier proteins } \\
\text { Contains a } \beta \text {-grasp fold }\end{array}$ \\
\hline \multicolumn{5}{|l|}{ Putative UBLs } \\
\hline BUBL1, BUBL2 & Variable (up to $80 \%$ ) & $\mathrm{NI}$ & $\mathrm{NI}$ & Putative autoprocessed proteins in ciliates \\
\hline UBL-1 & 40 & $\mathrm{NI}$ & $\mathrm{NI}$ & A precursor to ribosomal proteins in nematodes \\
\hline SF3A120 & 30 & $\mathrm{NI}$ & $\mathrm{NI}$ & $\begin{array}{l}\text { UBL domain at } \mathrm{C} \text { terminus } \\
\text { No data about conjugation }\end{array}$ \\
\hline $\begin{array}{l}\text { Oligoadenylate } \\
\text { synthetase }\end{array}$ & 30 and $42^{\dagger}$ & $\mathrm{NI}$ & $\mathrm{NI}$ & $\begin{array}{l}\text { UBL domain at } \mathrm{C} \text { terminus } \\
\text { No data about conjugation }\end{array}$ \\
\hline \multicolumn{5}{|c|}{$\begin{array}{l}\text { * UBLs are listed as the yeast (Saccharomyces cerevisiae) symbol if the UBL is present in yeast, otherwise vertebrate symbols are listed. } \\
\text { Known vertebrate orthologues with symbols that differ from yeast proteins are listed in parentheses. SAMP1 and SAMP2 are proteins in } \\
\text { archaeal. For E1s and E2s, yeast symbols are listed if the protein is found in yeast. }\end{array}$} \\
\hline \multicolumn{5}{|c|}{ Data in this table is adapted from the article published by Mark Hochstrasser (2009). } \\
\hline
\end{tabular}

Urm1 is the most ancient UBL and plays a number of important roles in yeast bioprocesses (Table 1) such as budding (Goehring et al., 2003b), nutrient sensing (RubioTexeira, 2007), high temperature sensitivity (Furukawa et al., 2000), antioxidant stress response (Goehring et al., 2003a), and post-translational modification of the elongator subunit (Fichtner et al., 2003). Like UBLs, Urm1 also contains the Cterminal diglycine and $\beta$-grasp-fold structural motif. Previous studies have demonstrated that it can be covalently conjugated to a single substrate, Ahp1p, in budding yeast, mediated by the E1-like protein Uba4 (Furukawa et al., 2000; Goehring et al., 2003a). Recently, Van der Veen et al. (Van der Veen et al., 2011) significantly expanded this conclusion by revealing the specific oxidative stress-induced process of Urm1-protein conjugation in both yeast and human cells, and detected the 21 human proteins modified by Urm1 in response to oxidative stress. These include proteins involved in the Urm1/Ub pathway, nuclear transport, RNA binding and processing, oxidative stress, and tRNA modification (Table 2). Through a series of biochemical experiments, they confirmed a covalent linkage between Urm1 and the lysine residues of its protein substrates. These linkages are largely resistant to the reducing agents DTT and hydroxylamine, indicating the high probability that they are isopeptide bonds. Based on these experimental results, the function of Urm1 as a sulfur carrier is directly coupled to its functions as a ubiquitin-like modifier. Urm1 seems to occupy an important place in the evolutionary link between prokaryotic sulfur carrier proteins and eukaryotic UBLs (Fig. 1) and is regarded as a molecular fossil (Xu et al., 2006; Pedrioli et al., 2008; Petroski et al., 2011; Van der Veen et al., 2011).

\section{URM1 SYSTEM COMPONENTS}

To date, three independent yeast genome-wide screens have identified the five components responsible for the 2-thiolation modification of $\mathrm{mcm}^{5} \mathrm{~s}^{2} \mathrm{U}$ and protein urmylation, and these are Urm1, Uba4, Tum1, Ncs6, and Ncs2 (Lu et al., 2005, 2008; Nakai et al., 2008; Leidel et al., 2009). The cysteine desulfurase Nfs 1 is believed to be the upstream component in the system and acts as a sulfur donor (Nakai et al., 2004; Marelja et al., 2008), and its essential functions in cell survival 


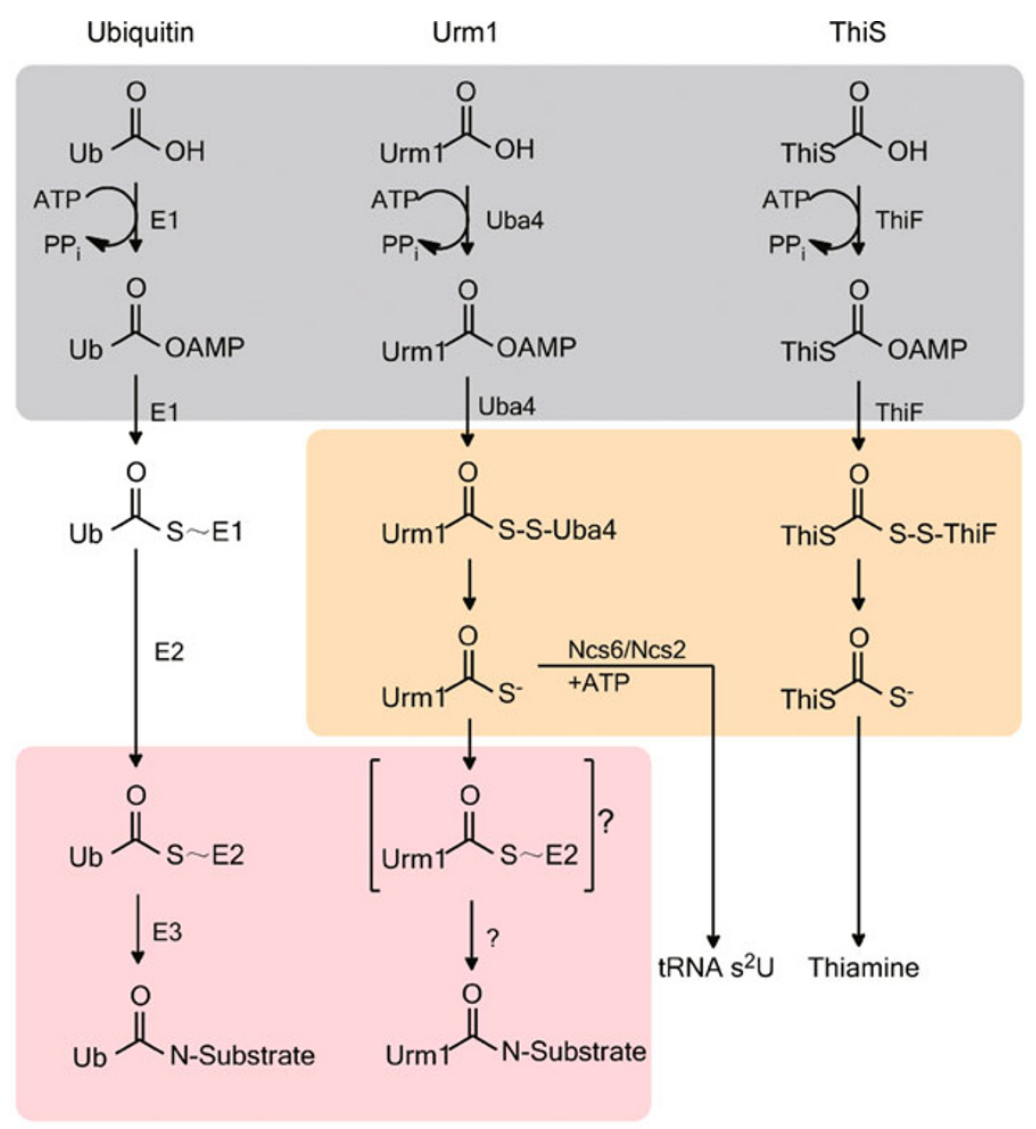

Figure 1. Similarities and differences between the ubiquitin, Urm1, and ThiS systems. The shaded areas show the overlapping parts of these pathways, revealing that Urm1 has features similar to ubiquitin and ThiS in the activation and conjugation process. This figure is a combination of the excellent work done by Petroski et al. (2011) and Pedrioli et al. (2008). No E2 or E3 for Urm1 has been detected, so the existence of an Urm1-E2 intermediate is still hypothetical. However, the isopeptides formed between Urm1 and the substrates have been proven by Van der Veen (2011).

may explain its nondetection in the screens (Nakai et al., 2001).

Among these proteins, Uba4 was discovered via a yeast two-hybrid screen using Urm1 as bait. Uba4 has a distinct sequence similarity to Uba1, an ubiquitin-activating enzyme. Furukawa et al. (Furukawa et al., 2000) demonstrated that Urm1 conjugation depends on the function of Uba4 as its E1like protein. They found that a DTT-sensitive bond is formed between Uba4 and Urm1, and deduced that this bond may also be a thioester, similar to other UBLs. This conclusion has been recently challenged by the discovery of the presence of the Urm1 C-terminal thiocarboxylate, which suggests that the Urm1-Uba4 complex is bound by a disulfide bridge (Pedrioli et al., 2008; Schmitz et al., 2008; Van der Veen et al., 2011). Uba4 has two conserved cysteines: Cys397 in the C-terminal rhodanese-like domain (RLD) and Cys225 in the MoeB-like domain. Rhodaneses are widespread enzymes that catalyze the transfer of sulfane sulfur atoms from thiosulfate to cyanide and serve as versatile sulfur carriers (Bordo and Bork et al., 2002; Mueller, 2006). Several groups have demonstrated that the two Uba4 cysteines are essential for Urm1 thiocarboxylate formation and tRNA thiolation, but only Cys397 is essential for thiosulfate sulfurtransferase activity (Fig. 2A) (Schmitz et al., 2008; Hochstrasser, 2009; Noma et al., 2009).

Before activating Urm1, Uba4 needs to receive sulfur atoms from Nfs1 and Tum1. Noma et al. (2009) used a series of clever in vitro sulfur transfer assays and revealed that by using pyridoxal-5-phosphate (PLP) as a co-factor, Nfs1 accepts sulfur from cysteine to form a persulfide and subsequently transfers this bond to the rhodanese-like domain in Tum1 or Uba4. Sequence alignment has detected two rhodanese-like domains in Tum1, but site-directed mutagenesis demonstrates that only the Cys259 in the Cterminal RLD is essential for persulfide formation. The $\mathrm{N}$ terminal RLD seems inactive, with no conserved cysteines. Thus, Tum1 is believed to be a crucial Nfs1 activator as well as an optional persulfide carrier. Nfs 1 can directly transfer persulfide sulfurs to Uba4, and Tum1 can significantly enhance this transfer activity (Fig. 2A) (Bordo and Bork et al., 2002; Noma et al., 2009). 
A

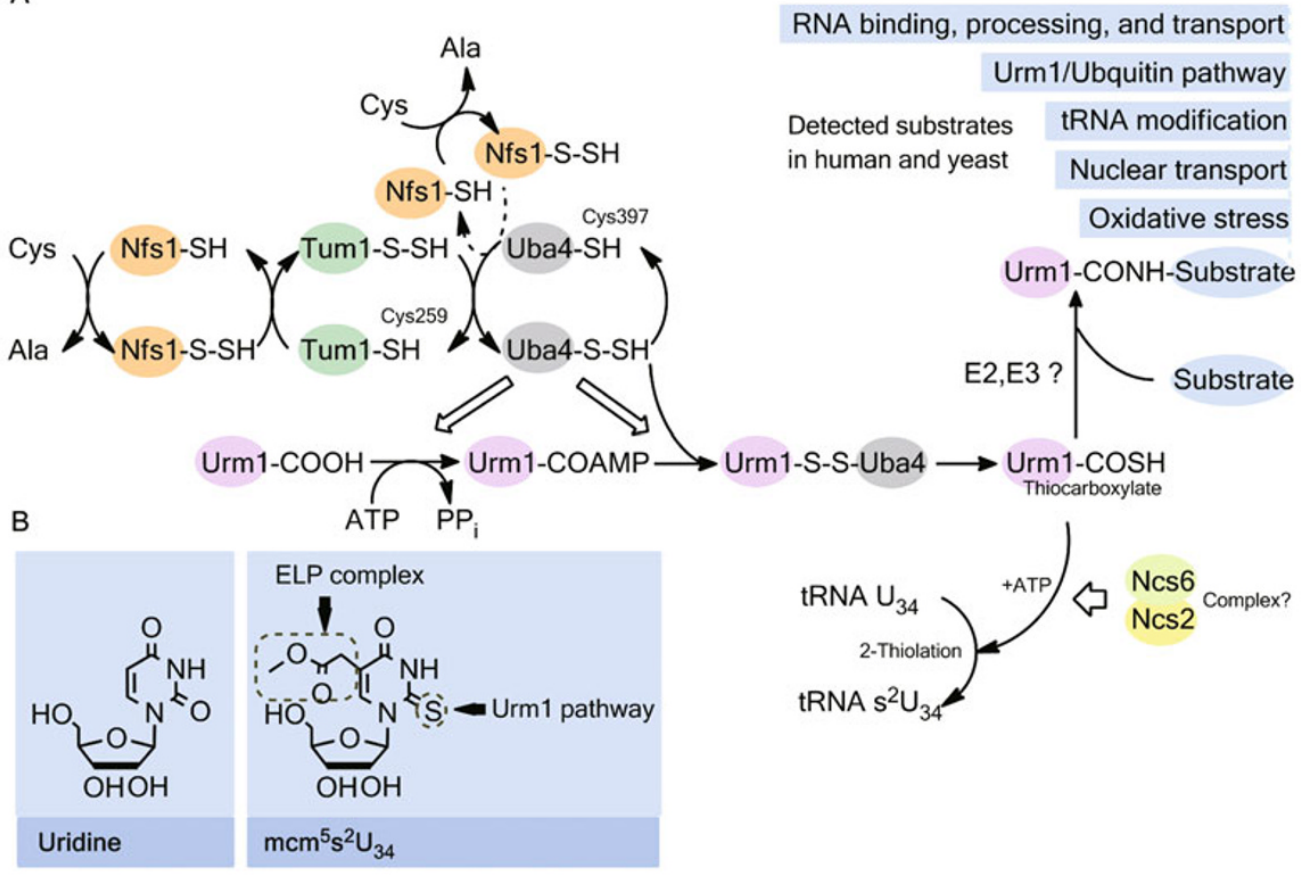

Figure 2. Urm1 pathway involved in the tRNA modification and protein conjugation processes. (A) Overview of the sulfur flow process between the Urm1 system components for 2-thiouridine formation of $\mathrm{mcm}^{5} \mathrm{~s}^{2} \mathrm{U}_{34}$ and the Urm1 conjugation process with its detected substrates. (B) Structure of uridine and $\mathrm{mcm}^{5} \mathrm{~s}^{2} \mathrm{U}_{34}$. The Urm1 pathway is responsible for 2-thiolation, whereas the ELP complex is responsible for $\mathrm{mcm}^{5} \mathrm{U}_{34}$ modification. These pictures are adapted from the articles published by Pedrioli et al. (2008), Noma et al. (2009), and Van der Veen et al. (2011).

After receiving a sulfur atom from Nfs1 or Tum1, Uba4 activates Urm1 by forming an acyl adenylate intermediate at the Urm1 C-terminal, and then forms an acyl disulfide bond between Uba4 Cys397 and the Urm1 C-terminal glycine (Leidel et al., 2009; Noma et al., 2009; Van der Veen et al., 2011). Afterward, a thiocarboxylated Urm1 is released, which provides the necessary sulfur for subsequent tRNA modification or protein conjugation (Fig. 2A). The Urm1 C-terminal glycine is essential for thiocarboxylate formation, and this has been proven by analyzing Urm1 WT and Urm1 $\Delta G$ through a polyacrylamide gel supplemented with [( $\mathrm{N}$-acryloylamino)phenyl]mercuric chloride (APM-modified gels) (Van der Veen et al., 2011).

Ncs6 and Ncs2 belong to the PP-loop ATPases and are essential for the in vivo 2-thiolation modification of $\mathrm{mcm}^{5} \mathrm{~s}^{2} \mathrm{U}$, using Urm1-COSH as the substrate. Several articles have reported the essential role of Ncs6 and its homologues in the 2-thiolation of tRNA uridine residues of yeast, worms, and Thermus thermophilus (Shigi et al., 2006; Björk et al., 2007; Dewez et al., 2008). In vitro binding assay also reveals that recombinant MBP-Ncs6 can directly bind yeast tRNA, whereas MBP-Uba4 cannot (Leidel et al., 2009). The function of Nfs2 in this process remains unclear, because recombinant Ncs2 is insoluble and difficult to test in vitro. Dewez, Leidel, and their co-workers (Dewez et al., 2008; Leidel et al., 2009) proposed that the two ATPases may form a complex (Fig. 2A), but this has been challenged by Nakai and his co-workers (Nakai et al., 2008). To date, the specific protein that transfers sulfur atoms from the thiocarboxylated Urm1 has not been identified, and the mechanism behind Ncs6 and Ncs2 recognition of the specific tRNA and catalysis of 2-thiouridine formation has not been established (Noma et al., 2009).

\section{URM1 AS A PROTEIN MODIFIER}

As previously mentioned, the function of Uba4 as E1 in the Urm1 conjugation process has been clearly revealed. To date, no Urm1-specific Urm1-conjugating E2 enzyme, Urm1lignase enzyme $\mathrm{E} 3$, and deurmylation enzyme have been detected. Therefore, Hochstrasser (Hochstrasser, 2000) proposed that Uba4 may also function as an E2-like enzyme. However, this hypothesis is challenged by the recent discovery of an acyl-disulfide bond between Urm1 and the conserved cysteine of Uba4 (Schmitz et al., 2008), because a thioester linkage is typically formed in UBL E2 enzymes. Van der Veen et al. (2011) have proven that the C-terminal thiocarboxylated Urm1 is necessary for the conjugation process. No conjugation was detected when Urm1-COSH was replaced with EGFP-COSH, indicating that a thiocarboxylate intermediate is not enough for conjugation formation and 
Table 2 Human protein substrates uniquely modified by Urm1 in response to oxidative stress

\begin{tabular}{|c|c|c|c|c|}
\hline Reference & Protein name & Architecture & Diamide & $\mathrm{H}_{2} \mathrm{O}_{2}$ \\
\hline \multicolumn{5}{|c|}{ Urm1/Ub pathway } \\
\hline NP_660275 & ATP binding domain 3 & AANH-like II & + & + \\
\hline NP_055299 & Molybdenum cofactor synthesis protein 3 & E1-like NBD, RHD & + & + \\
\hline NP_006304 & Ubiquitin specific protease 15 & DUSP domain & - & + \\
\hline NP_060414 & Ubiquitin specific protease 47 & $\mathrm{UCH}$ & + & + \\
\hline NP_062538 & E3 ubiquitin-protein ligase BRE1A & RING-finger & - & + \\
\hline \multicolumn{5}{|c|}{ tRNA modification } \\
\hline NP_060225 & 5-methycytoisine methyltransferase & NOL1/NOP2/sun family & + & + \\
\hline NP_003631 & Elongator complex protein 1 & IKI3 family & - & + \\
\hline \multicolumn{5}{|c|}{ Nuclear transport } \\
\hline NP_001307 & Chromosome segregation 1-like & CAS_CSE1 & - & + \\
\hline NP_002874 & Ran GTPase-activating protein 1 & LRR_RI & - & + \\
\hline NP_006353 & Nuclear RNA export factor 1 isoform 1 & NTF2 & - & + \\
\hline NP_056046 & Nuclear pore complex protein Nup160 & Nucleoporin Nup160 & - & + \\
\hline \multicolumn{5}{|c|}{ RNA binding, processing, and transport } \\
\hline NP_056455 & Serpine 1 mRNA-binding protein 1 & HABP4_PAI-RBP1 family & - & + \\
\hline NP_001410 & ELAV-like protein 1 & RNA recognition motif & + & - \\
\hline NP_002902 & Regulator of nonsense transcripts 1 & UPF1_Zn_bind family & + & - \\
\hline NP_006436 & Splicing factor Prp8 & MPN_PRP8 & + & - \\
\hline NP_004388 & ATP-dependent RNA helicase p54 & DEAD-box helicases & - & + \\
\hline \multicolumn{5}{|l|}{ Oxidative stress } \\
\hline NP_000604 & Hemopexin precursor & Hemopexin-like repeats & + & - \\
\hline NP_001748 & Carbonyl reductase [NADPH] 1 & NADB_Rossmann & - & + \\
\hline \multicolumn{5}{|l|}{ Miscellaneous } \\
\hline NP_858059 & O-GIcNAc transferase $\mathrm{p} 110$ subunit & TPR & + & - \\
\hline NP_001054 & Serotransferrin precursor & Transferrin super family & + & - \\
\hline NP_005555 & Migration-stimulating factor inhibitor & Lipocalin super family & + & - \\
\hline
\end{tabular}

that the recognition of protein substrates by Urm1 is specific; however, the mechanism for this remains unclear at present.

In yeast, the thioredoxin peroxidase protein Ahp1, which plays an essential role in cellular response to oxidative stress (Jeong et al., 1999; Lee et al., 1999), is the only known Urm1 substrate (Goehring et al., 2003a). Recently, Van der Veen et al. (2011) found that oxidative stress can induce Urm1 substrate conjugation in both yeast and human cells (Table 2). Interestingly, both $\mathrm{H}_{2} \mathrm{O}_{2}$ and diamide can stimulate the conjugation in vivo, but their substrates are different. In the present article, 21 proteins have been identified as Urm1 uniquely modified substrates induced by either $\mathrm{H}_{2} \mathrm{O}_{2}$ or diamide (Fig. 2A; Table 2). Site-directed mutagenesis assay clearly reveals that Urm1 is attached to the specific lysine residues of the substrates. However, the mechanism of this process remains to be determined. Polyurmylation has not been observed among these linkages, and all tested substrates appear to be modified by a single Urm1 molecule, despite the several exposed lysine residues on Urm1 itself (Singh et al., 2005; Xu et al., 2006; Yu and Zhou, 2008).

The known urmylation substrates include two members of the Urm1 pathway, namely, MOCS3 (Uba4 homologue in Homo sapiens) and ATPD3 (Ncs6 homologue in Homo sapiens). Why Urm1 modifies the components in its own pathway in response to oxidative stress is still unclear (Table 2). Furthermore, two deubiquitinating enzymes, USP15 and USP47, are modified by Urm1; however, immunoblotting assay did not detect their deurmylation activity. Furthermore, several proteins related to nuclear transport, tRNA modification, and RNA processing have been identified, such as the cellular apoptosis susceptibility protein (Behrens et al., 2003), but the functions of these Urm1 modifications remain unknown (Van der Veen et al., 2011). Observation reveals that, unlike ubiquitin, Urm1 does not appear to have a role in targeting proteins for degradation. Although Urm1 may regulate post-translational modification by modifying 
deubiquitinating enzymes, or prevent the translocation of CAS to the nucleus, evidence for this is still lacking (Petroski et al., 2011).

\section{URM1 AS A SULFUR CARRIER IN tRNA MODIFICATION}

The Urm1 pathway plays an essential role in the 2-thiolation modification of certain cytosolic tRNA. The sulfur transfer process in the pathway has already been described in detail. tRNA has four canonical bases-adenosine, guanosine, cytidine, and uridine-and also has more than 70 kinds of known post-transcriptional modifications (Huang et al., 2008). These modifications can stabilize tRNA, enhance the accuracy of codon binding, reduce the conformational dynamics (Wang et al., 2007; Agris, 2008), and slightly regulate tRNA interactions with $\mathrm{mRNA}$ and the ribosome (Sen and Ghosh, 1976; Björk et al., 2007; Johansson et al., 2008). Among these, the wobble modification of uridine, $\mathrm{mcm}^{5} \mathrm{~s}^{2} \mathrm{U}$, is required for the proper decoding of NNR codons in eukaryotes (Noma et al., 2009). This modification is formed via two steps: the oxygen atom at position 2 of the wobble uridine is substituted by a sulfur atom provided by the Urm1 pathway; and position 5 of the uridine residue is modified with a methoxy-carbonyl-methyl by the six-subunit elongator protein (ELP) complex (Fig. 2B) (Svejstrup, 2007; Pedrioli et al., 2008).

Thiocarboxylated Urm1 not only serves as a sulfur donor in the tRNA thiolation process, but also functions as a protein modifier in response to oxidative stress. So what are the relationships between these two functions? Urm1-mediated tRNA modification may be regulated by urmylation of the effective substrate (Huang et al., 2008). Interestingly, an essential part of the ELP complex, the elongator complex protein 1 , is among the recently identified urmylation substrates in the presence of $\mathrm{H}_{2} \mathrm{O}_{2}$ (Van der Veen et al., 2011). This observation suggests a link between tRNA modification and protein urmylation, which needs further evidence.

\section{FUTURE PERSPECTIVES}

The past years have witnessed rapid progress in Urm1 pathway research, such as revealing its function as a sulfur carrier and detecting new substrates for Urm1 conjugation. However, several important questions still need to be answered.

First, questions concern the components involved in the tRNA- and protein-modifying branches of the Urm1 system. To date, no Urm1-specific conjugating and ligating enzymes (E2, E3) have been found. How does substrate recognition take place in the absence of a ligase? (Pedrioli et al., 2008) Biochemical experiments have demonstrated that the Cterminal thiocarboxylated EGFP is not sufficient to induce conjugation formation. In addition, given that Urm1 modifies the specific sites in a limited number of proteins, what determines the substrate recognition mechanism of Urm1 remains unclear. Furthermore, we still do not know whether the deurmylation process exists. Although two deubiquitinating enzymes, USP15 and USP47, are modified by Urm1, the author cannot find their activities in reversing urmylation (Van der Veen et al., 2011). Recent findings have revealed the necessary roles of Ncs2 and Ncs6 in the tRNA modification process, but we still do not know how they recognize specific tRNA and catalyze the reaction, and whether they can influence the urmylation level of proteins (Björk et al., 2007). Structural approaches may solve these questions by providing molecular models for these enzymes.

Questions have also been raised on the role of tRNA thiomodification: we still do not know the effect of the Urm1 pathway on protein translation. Translational efficiency and fidelity can be reduced by oxidative stress, which causes tRNA misacylation and impairs the editing activity of threonyltRNA synthetase, the crucial enzymes in genetic code translation (Netzer et al., 2009; Ling and Söll, 2010). Thus, Urm1 may act as an important post-translational modifier that alters the balance between tRNA thiolation and protein modification under oxidative stress.

In addition, components in the Urm1 system may be potential drug candidates, because they are essential for tRNA thiolation but are not crucial genes in yeast. Some retroviruses, such as the human immunodeficiency virus type 1 (HIV-1), use the cellular tRNA (Lys 3 ) as a primer for reverse transcription (Abbink and Berkhout, 2008). In vitro assay reveals that the AAAA/Umcm ${ }^{5} \mathrm{~s}^{2} \mathrm{UUU}$ loop-loop interaction is essential and conserved in the reverse transcription of HIV-1 isolates, and that dethiolation of the modified nucleotide $\mathrm{mcm}^{5} \mathrm{~s}^{2} \mathrm{U}$ at position 34 of tRNA3Lys strongly destabilizes this interaction (Isel et al., 1993, 1996). Therefore, further studies on the relevance between the Urm1 pathway and such diseases are needed.

The third question focuses on the function of Urm1 modification during oxidative stress. Based on observations, Urm1 does not appear to have a role in targeting proteins for degradation, and no polyurmylation has been detected. Why its E1 MOCS3, two deubiquitinating enzymes, CAS, and some other important enzymes are modified still remains unclear, because no change was found in the activity of these enzymes after Urm1 conjugation (Petroski et al., 2011; Van der Veen et al., 2011). Although these questions remain unanswered, we now clearly know that Urm1 is an important protein modifier that shares similar features with both sulfur carriers and UBLs.

\section{ABBREVIATIONS}

Ahp1, alkyl hydroperoxide reductase 1; ATPD3, ATP-binding domain 3; CAS, chromosome segregation 1-like protein; DTT, dithiothreitol; EGFP, enhanced green fluorescence protein; ELP, elongator protein; 
MBP, maltose-binding protein; $\mathrm{mcm}^{5} \mathrm{~s}^{2} \mathrm{U}, 5$-methoxycarbonylmethyl2-thiouridine; MoaD, molybdopterin synthase subunit; MOCS3, molybdenum cofactor synthesis protein $3 ; \mathrm{Ncs} 6 / \mathrm{Ncs} 2$, needs cla4 to survive protein 6/2; Nfs1, NIFS-like protein 1; PLP, pyridoxal-5phosphate; RLD, rhodanese-like domain; ThiS, thiamin biosynthesis; Tum1, thiouridine modification 1; Uba4, ubiquitin-like protein activating protein 4; UBLs, ubiquitin-like proteins; Urm1, ubiquitin-related modifier 1; USP15/USP47, ubiquitin-specific protease 15/47

\section{REFERENCES}

Abbink, T.E.M., and Berkhout, B. (2008). HIV-1 reverse transcription initiation: a potential target for novel antivirals? Virus Res 134, 4-18.

Agris, P.F. (2008). Bringing order to translation: the contributions of transfer RNA anticodon-domain modifications. EMBO Rep 9, $629-635$.

Behrens, P., Brinkmann, U., and Wellmann, A. (2003). CSE1L/CAS: its role in proliferation and apoptosis. Apoptosis 8, 39-44.

Björk, G.R., Huang, B., Persson, O.P., and Byström, A.S. (2007). A conserved modified wobble nucleoside $(\mathrm{mcm} 5 \mathrm{~s} 2 \mathrm{U})$ in lysyl-tRNA is required for viability in yeast. RNA 13, 1245-1255.

Bordo, D., and Bork, P. (2002). The rhodanese/Cdc25 phosphatase superfamily. Sequence-structure-function relations. EMBO Rep 3, 741-746.

Ciechanover A., Heller H., Katzetzion R., Hershko A. (1981). Activation of the Heat-Stable Polypeptide of the Atp-Dependent Proteolytic System. Proc Natl Acad Sci U S A 78, 761-765.

Dewez, M., Bauer, F., Dieu, M., Raes, M., Vandenhaute, J., and Hermand, D. (2008). The conserved Wobble uridine tRNA thiolase Ctu1-Ctu2 is required to maintain genome integrity. Proc Natl Acad Sci U S A 105, 5459-5464.

Fichtner, L., Jablonowski, D., Schierhorn, A., Kitamoto, H.K., Stark, M.J.R., and Schaffrath, R. (2003). Elongator's toxin-target (TOT) function is nuclear localization sequence dependent and suppressed by post-translational modification. Mol Microbiol 49, 1297-1307.

Furukawa, K., Mizushima, N., Noda, T., and Ohsumi, Y. (2000). A protein conjugation system in yeast with homology to biosynthetic enzyme reaction of prokaryotes. J Biol Chem 275, 7462-7465.

Goehring, A.S., Rivers, D.M., and Sprague, G.F. Jr. (2003a). Attachment of the ubiquitin-related protein Urm1p to the antioxidant protein Ahp1p. Eukaryot Cell 2, 930-936.

Goehring, A.S., Rivers, D.M., and Sprague, G.F. Jr. (2003b). Urmylation: a ubiquitin-like pathway that functions during invasive growth and budding in yeast. Mol Biol Cell 14, 4329 4341.

Haas, A.L., Warms, J.V.B., Hershko, A., and Rose, I.A. (1982). Ubiquitin-activating enzyme. Mechanism and role in proteinubiquitin conjugation. J Biol Chem 257, 2543-2548.

Hershko, A., Ciechanover, A., and Varshavsky, A. (2000). The ubiquitin system. Nat Med 6, 1073-1081.

Hochstrasser, M. (2000). Evolution and function of ubiquitin-like protein-conjugation systems. Nat Cell Biol 2, E153-E157.

Hochstrasser, M. (2009). Origin and function of ubiquitin-like proteins. Nature 458, 422-429.

Huang, B., Lu, J., and Byström, A.S. (2008). A genome-wide screen identifies genes required for formation of the wobble nucleoside 5-methoxycarbonylmethyl-2-thiouridine in Saccharomyces cerevisiae. RNA 14, 2183-2194.

Humbard, M.A., Miranda, H.V., Lim, J.M., Krause, D.J., Pritz, J.R., Zhou, G.Y., Chen, S.X., Wells, L., and Maupin-Furlow, J.A. (2010). Ubiquitin-like small archaeal modifier proteins (SAMPs) in Haloferax volcanii. Nature 463, 54-60.

Isel, C., Lanchy, J.M., Le Grice, S.F.J., Ehresmann, C., Ehresmann, B., and Marquet, R. (1996). Specific initiation and switch to elongation of human immunodeficiency virus type 1 reverse transcription require the post-transcriptional modifications of primer tRNA3Lys. EMBO J 15, 917-924.

Isel, C., Marquet, R., Keith, G., Ehresmann, C., and Ehresmann, B. (1993). Modified nucleotides of tRNA(3Lys) modulate primer/ template loop-loop interaction in the initiation complex of HIV-1 reverse transcription. J Biol Chem 268, 25269-25272.

lyer, L.M., Burroughs, A.M., and Aravind, L. (2006). The prokaryotic antecedents of the ubiquitin-signaling system and the early evolution of ubiquitin-like beta-grasp domains. Genome Biol 7, R60.

Jeong, J.S., Kwon, S.J., Kang, S.W., Rhee, S.G., and Kim, K. (1999). Purification and characterization of a second type thioredoxin peroxidase (type II TPx) from Saccharomyces cerevisiae. Biochemistry 38, 776-783.

Johansson, M.J.O., Esberg, A., Huang, B., Björk, G.R., and Byström, A.S. (2008). Eukaryotic wobble uridine modifications promote a functionally redundant decoding system. Mol Cell Biol 28, 3301-3312.

Lake, M.W., Wuebbens, M.M., Rajagopalan, K.V., and Schindelin, H. (2001). Mechanism of ubiquitin activation revealed by the structure of a bacterial MoeB-MoaD complex. Nature 414, 325-329.

Lee, J., Spector, D., Godon, C., Labarre, J., and Toledano, M.B. (1999). A new antioxidant with alkyl hydroperoxide defense properties in yeast. J Biol Chem 274, 4537-4544.

Leidel, S., Pedrioli, P.G.A., Bucher, T., Brost, R., Costanzo, M., Schmidt, A., Aebersold, R., Boone, C., Hofmann, K., and Peter, M. (2009). Ubiquitin-related modifier Urm1 acts as a sulphur carrier in thiolation of eukaryotic transfer RNA. Nature 458, 228-232.

Ling, J.Q., and Söll, D. (2010). Severe oxidative stress induces protein mistranslation through impairment of an aminoacyl-tRNA synthetase editing site. Proc Natl Acad Sci U S A 107, 4028- 4033.

Lu, J., Esberg, A., Huang, B., and Byström, A.S. (2008). Kluyveromyces lactis gamma-toxin, a ribonuclease that recognizes the anticodon stem loop of tRNA. Nucleic Acids Res 36, 1072-1080.

Lu, J., Huang, B., Esberg, A., Johansson, M.J.O., and Byström, A.S. (2005). The Kluyveromyces lactis gamma-toxin targets tRNA anticodons. RNA 11, 1648-1654.

Marelja, Z., Stöcklein, W., Nimtz, M., and Leimkühler, S. (2008). A novel role for human Nfs1 in the cytoplasm: Nfs1 acts as a sulfur donor for MOCS3, a protein involved in molybdenum cofactor biosynthesis. J Biol Chem 283, 25178-25185.

Miranda, H.V., Nembhard, N., Su, D., Hepowit, N., Krause, D.J., Pritz, J.R., Phillips, C., Söll, D., and Maupin-Furlow, J.A. (2011). E1- and ubiquitin-like proteins provide a direct link between protein conjugation and sulfur transfer in archaea. Proc Natl Acad Sci U S A 108, 4417-4422.

Mueller, E.G. (2006). Trafficking in persulfides: delivering sulfur in biosynthetic pathways. Nat Chem Biol 2, 185-194.

Nakai, Y., Nakai, M., and Hayashi, H. (2008). Thio-modification of yeast cytosolic tRNA requires a ubiquitin-related system that 
resembles bacterial sulfur transfer systems. J Biol Chem 283, 27469-27476.

Nakai, Y., Nakai, M., Hayashi, H., and Kagamiyama, H. (2001). Nuclear localization of yeast Nfs $1 \mathrm{p}$ is required for cell survival. $\mathrm{J}$ Biol Chem 276, 8314-8320.

Nakai, Y., Umeda, N., Suzuki, T., Nakai, M., Hayashi, H., Watanabe, K., and Kagamiyama, H. (2004). Yeast Nfs $1 \mathrm{p}$ is involved in thiomodification of both mitochondrial and cytoplasmic tRNAs. J Biol Chem 279, 12363-12368.

Netzer, N., Goodenbour, J.M., David, A., Dittmar, K.A., Jones, R.B., Schneider, J.R., Boone, D., Eves, E.M., Rosner, M.R., Gibbs, J.S., et al. (2009). Innate immune and chemically triggered oxidative stress modifies translational fidelity. Nature 462, 522-526.

Noma, A., Sakaguchi, Y., and Suzuki, T. (2009). Mechanistic characterization of the sulfur-relay system for eukaryotic 2thiouridine biogenesis at tRNA wobble positions. Nucleic Acids Res 37, 1335-1352.

Pedrioli, P.G.A., Leidel, S., and Hofmann, K. (2008). Urm1 at the crossroad of modifications. 'Protein Modifications: Beyond the Usual Suspects' Review Series. EMBO Rep 9, 1196-1202.

Petroski, M.D., Salvesen, G.S., and Wolf, D.A. (2011). Urm1 couples sulfur transfer to ubiquitin-like protein function in oxidative stress. Proc Natl Acad Sci U S A 108, 1749-1750.

Pickart, C.M., and Fushman, D. (2004). Polyubiquitin chains: polymeric protein signals. Curr Opin Chem Biol 8, 610-616.

Rubio-Texeira, M. (2007). Urmylation controls Nil1p and GIn3pdependent expression of nitrogen-catabolite repressed genes in Saccharomyces cerevisiae. FEBS Lett 581, 541-550.

Schlieker, C.D., Van der Veen, A.G., Damon, J.R., Spooner, E., and Ploegh, H.L. (2008). A functional proteomics approach links the ubiquitin-related modifier Urm1 to a tRNA modification pathway. Proc Natl Acad Sci U S A 105, 18255-18260.

Schmitz, J., Chowdhury, M.M., Hänzelmann, P., Nimtz, M., Lee, E.Y., Schindelin, H., and Leimkühler, S. (2008). The sulfurtransferase activity of Uba4 presents a link between ubiquitin-like protein conjugation and activation of sulfur carrier proteins. Biochemistry 47, 6479-6489.

Sen, G.C., and Ghosh, H.P. (1976). Role of modified nucleosides in tRNA: effect of modification of the 2-thiouridine derivative located at the 5 '-end of the anticodon of yeast transfer RNA Lys2. Nucleic Acids Res 3, 523-535.

Shigi, N., Sakaguchi, Y., Suzuki, T., and Watanabe, K. (2006). Identification of two tRNA thiolation genes required for cell growth at extremely high temperatures. J Biol Chem 281, 14296-14306.

Singh, S., Tonelli, M., Tyler, R.C., Bahrami, A., Lee, M.S., and Markley, J.L. (2005). Three-dimensional structure of the AAH26994.1 protein from Mus musculus, a putative eukaryotic Urm1. Protein Sci 14, 2095-2102.

Sun, L.J., and Chen, Z.J. (2004). The novel functions of ubiquitination in signaling. Curr Opin Cell Biol 16, 119-126.

Svejstrup, J.Q. (2007). Elongator complex: how many roles does it play? Curr Opin Cell Biol 19, 331-336.

Ulrich, H.D. (2002). Degradation or maintenance: actions of the ubiquitin system on eukaryotic chromatin. Eukaryot Cell 1, 1-10.

Van der Veen, A.G., Schorpp, K., Schlieker, C., Buti, L., Damon, J.R., Spooner, E., Ploegh, H.L., and Jentsch, S. (2011). Role of the ubiquitin-like protein Urm1 as a noncanonical lysine-directed protein modifier. Proc Natl Acad Sci U S A 108, 1763-1770.

Wang, C.Y., Xi, J., Begley, T.P., and Nicholson, L.K. (2001). Solution structure of ThiS and implications for the evolutionary roots of ubiquitin. Nat Struct Biol 8, 47-51.

Wang, X.J., Yan, Q.F., and Guan, M.X. (2007). Deletion of the MTO2 gene related to $t R N A$ modification causes a failure in mitochondrial RNA metabolism in the yeast Saccharomyces cerevisiae. FEBS Lett 581, 4228-4234.

Xi, J., Ge, Y., Kinsland, C., McLafferty, F.W., and Begley, T.P. (2001). Biosynthesis of the thiazole moiety of thiamin in Escherichia coli: identification of an acyldisulfide-linked protein-protein conjugate that is functionally analogous to the ubiquitin/E1 complex. Proc Natl Acad Sci U S A 98, 8513-8518.

Xu, J.J., Zhang, J.H., Wang, L., Zhou, J., Huang, H.D., Wu, J.H., Zhong, Y., and Shi, Y.Y. (2006). Solution structure of Urm1 and its implications for the origin of protein modifiers. Proc Natl Acad Sci U S A 103, 11625-11630.

Yu, J., and Zhou, C.Z. (2008). Crystal structure of the dimeric Urm1 from the yeast Saccharomyces cerevisiae. Proteins 71 , $1050-1055$. 